\title{
Automated Classification of Retinal Diseases in STARE Database
}

\author{
Tooba $^{1}$, Syed Omer Gilani ${ }^{1}$, Adeeb Shehzad ${ }^{1}$, Mohsin Jamil ${ }^{2}$, \\ Shafaq Mushtaq ${ }^{1}$, Sumbal Mushtaq ${ }^{1}$, Asim Waris ${ }^{1}$ \\ ${ }^{1}$ Department of Biomedical Engineering and Sciences \\ ${ }^{2}$ Department of Robotics and Artificial Intelligence \\ National University of Sciences and Technology (NUST), Islamabad, Pakistan \\ omer@smme.nust.edu.pk, tooba.shafa@yahoo.com, adeeb.shezad@gmail.com, \\ shiffa92@gmail.com, sumbly94@gmail.com ,asim.waris@smme.edu.pk
}

\begin{abstract}
Retinal diseases are identified by conducting different medical examinations primary of which are visual examinations. One of the key issues in visual diagnosis of diseases is the human error due to poor decision making, for that an upcoming inter-disciplinary technology named Computer Aided Medical Diagnostic System provides precise detection and prediction of disease. Automated image analysis methods are far more helpful for early identification and evaluation of disease as compared to cryptic and time taking manual techniques of digital medical imaging. This study aims to develop an automated method for identification of eye disorders that affect the human retina which if left unidentified may result in blindness due to delayed detection and analysis. Image data was acquired by publically available STARE Database having fundus images and by implementation of exclusion and inclusion criterion it was pre-processed on MATLAB. Initial pre-processing increased the significance of the data to be analyzed. From 186 images, 16 diseases and 22 features were deduced. A support vector machine classifier was used for automated identification and classification, resulting in an accuracy of $94 \%$ and specificity of $98 \%$. In the chosen technique sensitivity, specificity and accuracy of the results was affected by the problem of one-sided data. For the reduction of dimensionality of data (redundancy reduction) principal component analysis was employed. 5, 10 and 22 Principal components were obtained to reduce the amount of variables. PCA was performed prior to training of the $S V M$, results for different data dimensionality was compared for completeness.
\end{abstract}

Keywords: Image processing, support vector machine, principal component analysis, MATLAB, fundus images, retinal diseases

\section{Introduction}

Retina of human is composed cells known as rods and cones that are responsible for the image and color identification [1]. The neural layer connected to the retina through the optic nerve serves the most pivotal part in the human vision [2]. In the structure of the human eye, blood vessels, optic disc, fovea and macula are fundamental elements which form the retina. The focal point of the OD is also called the blind spot because it does not have any photoreceptor [3].

The macula lutea is an oval shaped dim spot around $0.4 \mathrm{~mm}$ in width located parallel to blind spot in eye. The center of the macula, about the size of a pin head is called Fovea Centralis and it has only cones to assist in sharp vision [4]. Most of the cones are present in macula and its thickness gradually reduces progressing towards the periphery [5].

Human retina undergoes several retinal deformities due to unhealthy living style, which influences the overall health of the eye. Abnormalities such as hemorrhages, macular degeneration, glaucoma and diabetic retinopathy [6] are a few of the many degenerative conditions of the retina [7]. A wide range of characteristic changes occur in the retina due to different diseases, a few of the symptoms are hemorrhages, cotton wool spots, exudates and micro aneurisms of the retina. 
A number of automated techniques are present in the latest research literature for identification of features of the human eye and their underlying abnormalities (Gharaibeh, 2016). Approached by Lee and Wang (1999) was introduced that addressed the problem of automatically detecting the quality of the fundus image [8]. Their approach starts from the perspective of addressing a pure signal by applying the global image intensity chart roughly by the Gaussian distribution. Nasr Al-Gharaibeh, Al-Huson (2015) suggested an approach that consists of pretreatment, vascular segmentation (FPCM), localization of necrosis, fovea removal, extraction and classification of malignant neural features [9]. Neuro-Fuzzy states as a hybrid of fuzzy logical and neural networks were employed in the design. Evaluations were performed utilizing MATLAB and the MESSIDOR database for their analysis that provides productive conclusions in vulnerability, quality, categorization, precision and reliability. Jing Wu et al. (2016) introduced a fully documented approach to detect fovea SD-OCT scans in a healthy and diseased macula [10]. This allows the use of the fovea as a key parameter in building a population reference frame to identify and exact key spatial temporal features from a large group of patients consisting of different time points, devices and imaging modalities. Priyanka B. Kale and Nitin Janwe (2017) proposed using an automated system to identify patients with diabetic retinopathy using fundus images [11].

The diagnosis and treatment related conclusions are easily and accurately drawn by the physician using automated medical image analysis. A number of automated techniques utilize machine learning and reinforcement learning as a primary solution for the decision and classification problems. Algorithms such as Support Vector Machines (SVM), Artificial Neural Networks, K-Nearest Neighbor and Naïve Bayes Classification are a few of the most popular techniques of diseases identification and classification.

This study was aimed to use publically available data in the STARE Database to perform the automated classification of retinal diseases using support vector machine (SVM) classifier (Figure 1). The Support vector machine (SVM) is used to perform supervised learning with an associated learning algorithm. This is a classification technique which was introduced by Boser et. al. in 1992 [12]. It can be readily employed to data with high-dimensionality such as that of the symptoms of retinal or any biological conditions.

\section{Methodology}

Publically accessible database of retinal image at Structured Analysis of the Retina (STARE) project was used to evaluate automated classification of retinal diseases. The initial idea was an image interpretation approach that differentiates the diseases of retina using fundus images. This contain colored images of retina obtained using 50 fundus camera that is of TRV (Topcon Corp., Tokyo, Japan) with $605 \times 700$ pixels resolution at 35 degree field. 397 images in 14 disease categories are present in database including cilio retinal artery occlusion (CRAO), emboli, branch retinal artery occlusion (BRAO), hemi-CRVO, proliferative diabetic retinopathy (PDR), arteriosclerotic retinopathy, central background diabetic retinopathy (BDR) (Singer et al., 1992), branch retinal vein occlusion (BRVO), choroidal neo-vascularization (CNV), hypertensive retinopathy, Coat's disease, retinal vein occlusion (CRVO), macroaneurism, and other retinal status. These other retinal statuses were further separately classified with new codes resulting in 41 diseases.

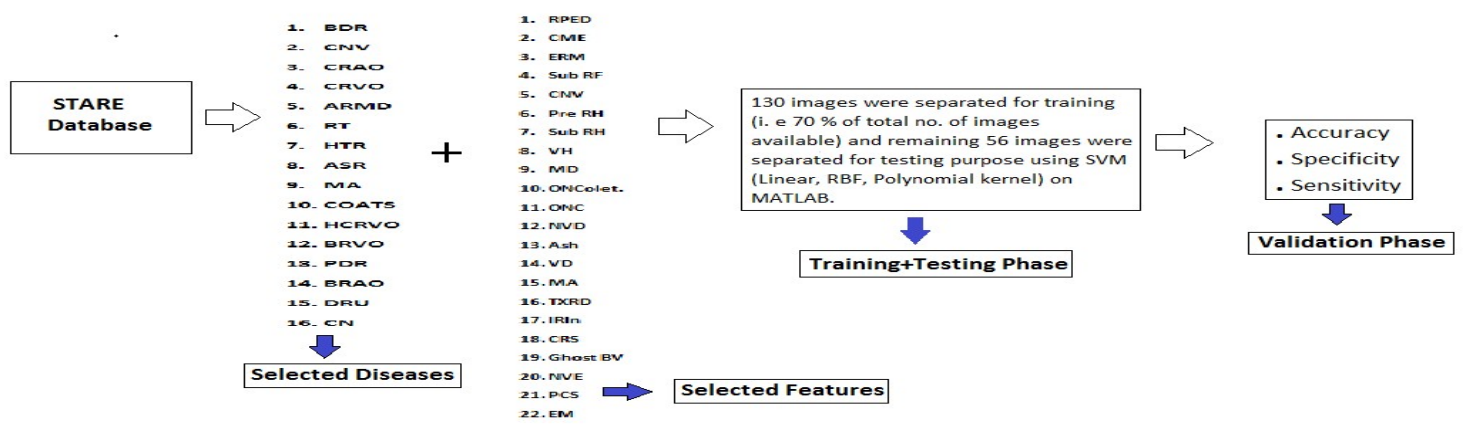


Figure 1: Proposed Procedure

Images were tallied for every disease in MATLAB and a reduction criterion was employed for diseases, features and images. Database contains 402 images, 41 diseases and 37 features.

Among 402 images only 311 images have valid information about features and diseases. A first criterion was the removal of diseases with less than 5 images. 91 images were removed due to improper diagnosis (according to the database), thus resulting in 21 diseases a first reduction, 2 categories (unknown

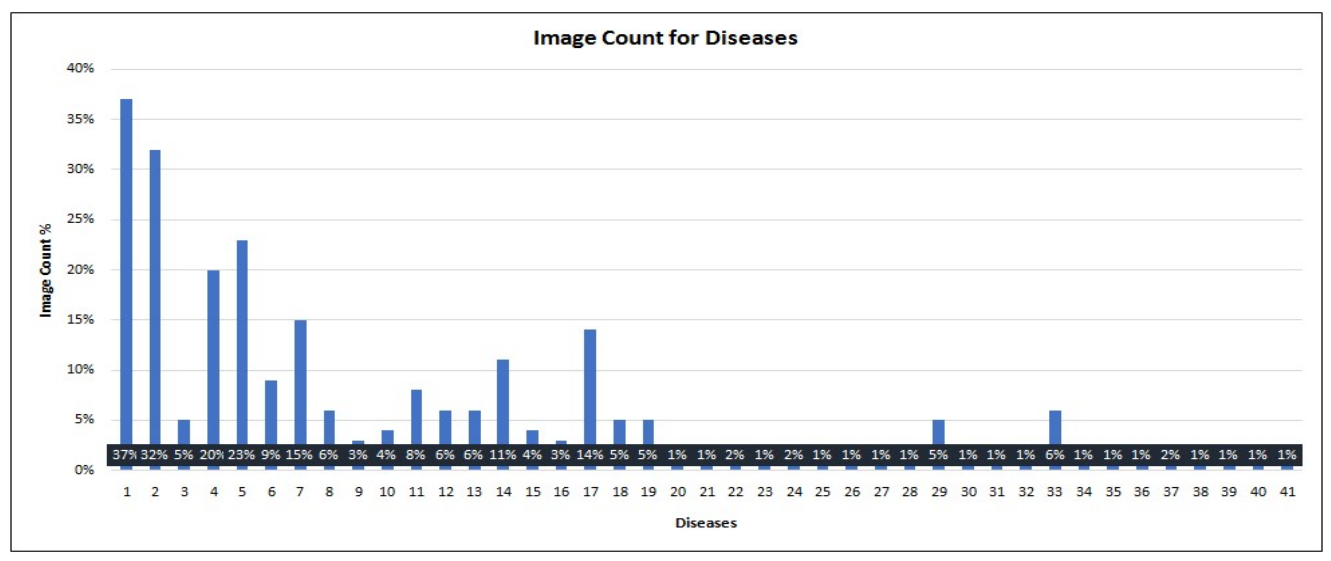

Figure 2: Total image count (\%) for each disease

diagnosis and choroidal haemangioma) were not used as recommended by the STARE project [13], leaving only 19 diseases, 37 features and 301 images in second reduction case.

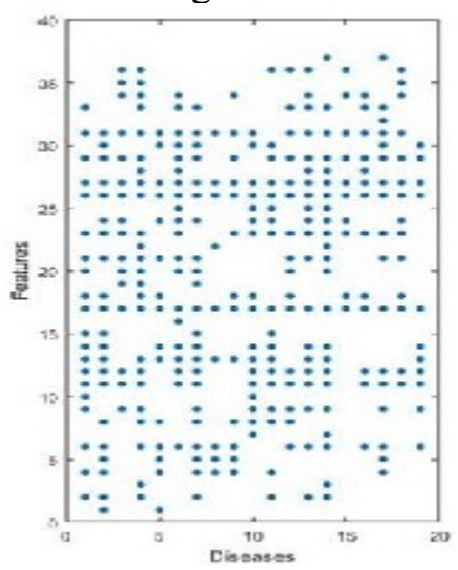

Figure 3: Diseases vs Features plot (19 diseases and 37 features)

MATLAB tool used for the reduction of images in database to satisfy the criteria of selection.

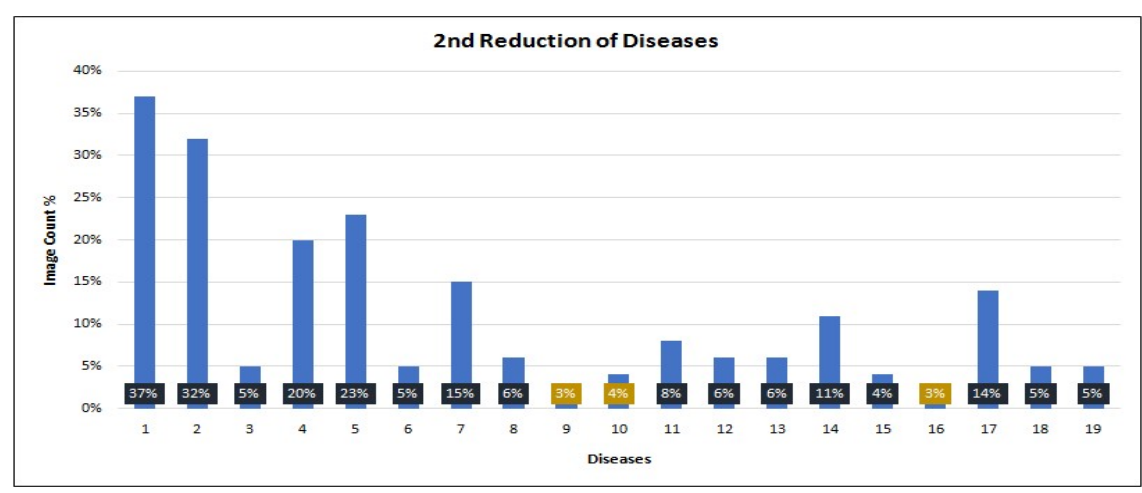

Figure 4: Reduced image count for each disease 
Afterwards the dataset was further reduced by applying a $50 \%$ criteria to 19 diseases eliminating 15 features and 112 images as a result we get 189 valid images for 22 features with 19 diseases.
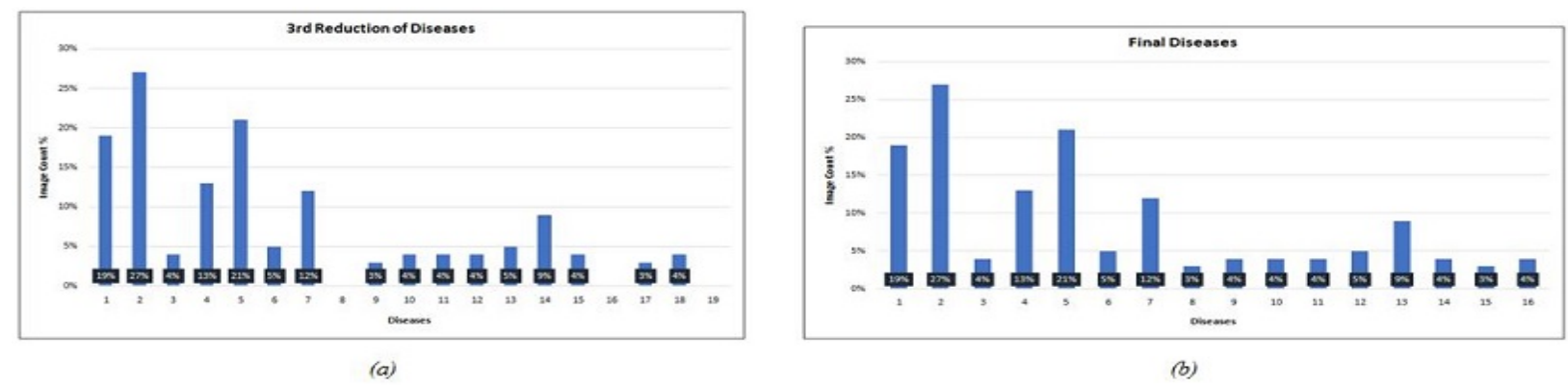

Figure 5: (a) Removal of reduced diseases ( 3 diseases containing $<5$ images),

(b) Final disease count (16 Disease, 22 Features and 186 Images)

The images which were removed in the above step also had some images of valid 19 diseases that causes skewed results for instance none of the 22 selected features were present however the disease was present in the image diagnosis. So a final reduction to fix the diseases was performed and images of 3 diseases were removed leaving valid 186 images and 16 diseases.

In order to utilize a Support Vector Machine the data was separated into training and testing samples. Biased random sampling was performed on the reduced data of 186 images to split into $70 \%$ training and 30\% testing data. The MATLAB Code was used for the biased random sampling of the data.

This data was split into training and testing samples through biased random sampling in the ascending order of positive samples of the diseases, min numbers of occurrences were for disease no. 15 (i.e. 5 positive samples). This ensured that the uneven distribution of the data was catered in the sampling process. Around $70 \%$ of the samples were sampled through biased random sampling for training and the remaining 30\% were separated for testing. Total 130 images were separated for training (i.e. $70 \%$ of total no. of images available) and remaining 56 images were separated for testing purpose.
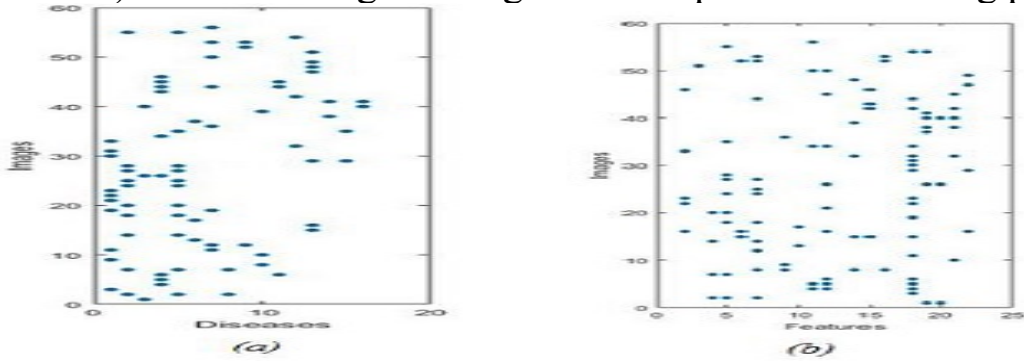

Figure 6: (a) Plot showing testing set of 16 Diseases vs 56 Images,

(b) Plot showing testing set of 22 Features vs 56 Images
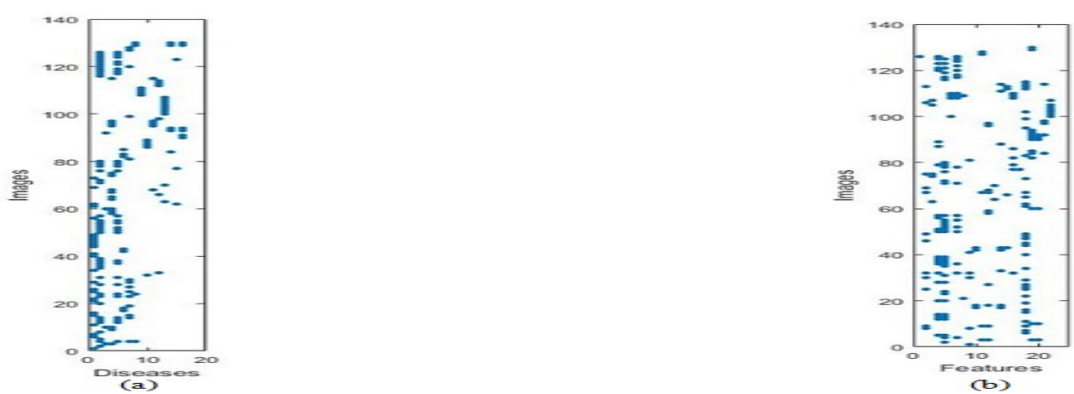

Figure 7 (a) Plot showing training set of 16 Diseases vs 130 Images,

(b) Plot showing training set of 16 Features vs 130 Images 
The figure 8 (a) below shows the split positive samples of data for all diseases in the available reduced data. The diseases with very less positive samples were prone to over-fitting in the case of true random sampling, hence biased random sampling was performed to minimize over-fitting.

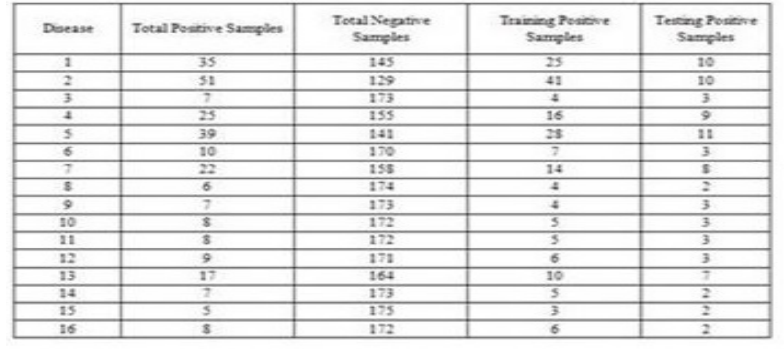

(a)

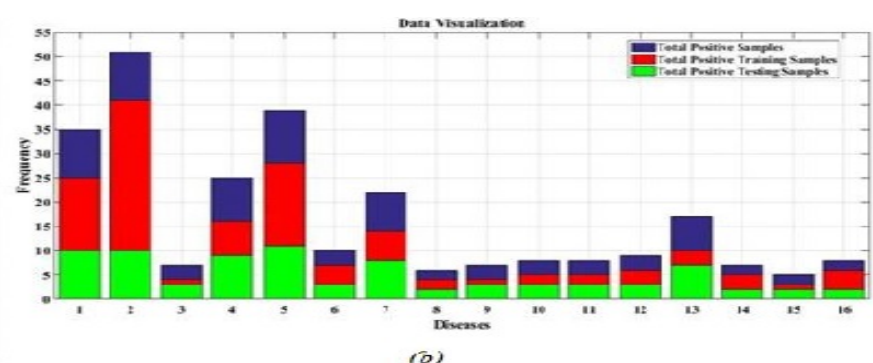

(o)

Figure 8 (a) Count of total, training and testing positive samples for each disease, (b) Representation of positive samples for training and testing (Blue: total positive samples. Red: total Positive training samples, Green: Total positive testing samples)

This biased random sampling was used because total number of positive samples per disease varies from 5 to 51 with normal random sampling all positive samples of some diseases were selected for training; while down to $50 \%$ of the positive samples of some diseases were selected for training. Biasing the random sampling ensures the diseases with least number of positive samples were treated first while ensuring random sampling for training. Figure 9 proves that biased random sampling (green line trend) is more closely related to $70 \%$ training set (blue reference line for $70 \%$ ) as compared to normal random sampling (red line trend).

Each sample point has 22 features and labels for presence (or absence) of 16 diseases. A total of 130

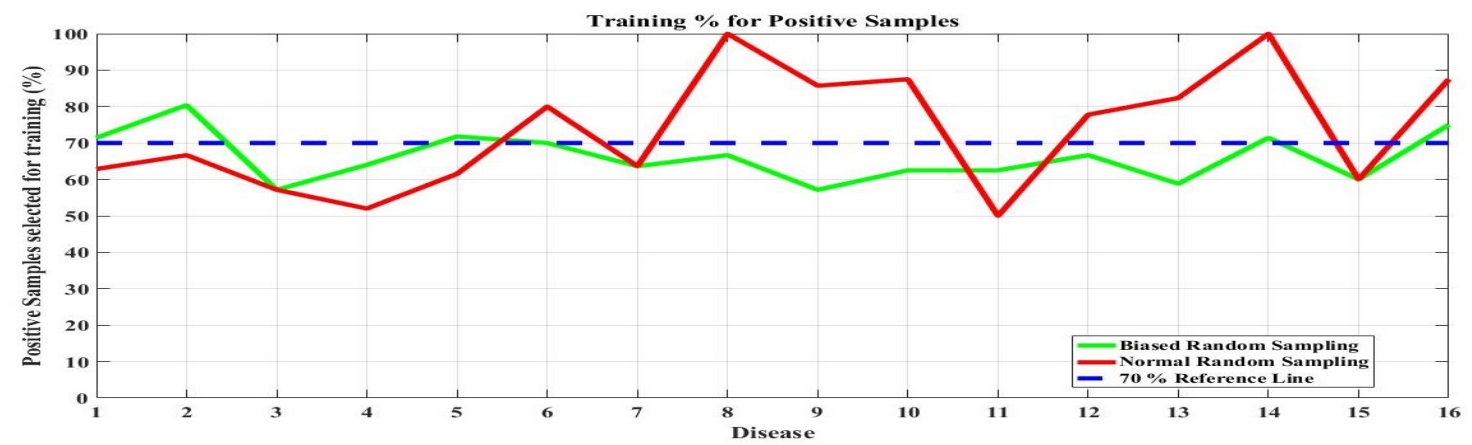

samples were used for training and 56 samples were used for testing the SVM algorithm.

Figure 9 Graph showing trend of biased random sampling

A Support Vector Machine (SVM) performs classification by finding the hyper plane that maximizes the

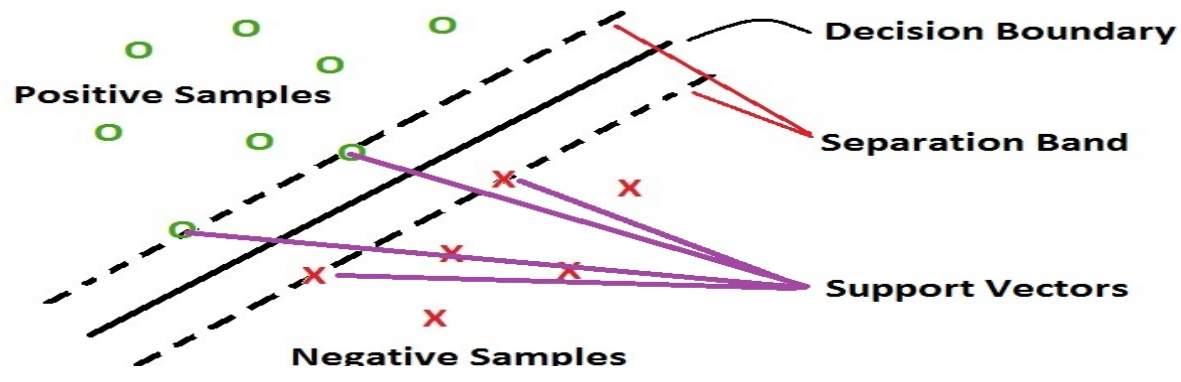

Figure 10: Basic Scheme of an SVM Classifier 
margin between the two classes [14]. The vectors (cases) that define the hyper plane are termed as the support vectors [15].

The kernel can be changed to incorporate data that is not linearly separable by a hyper plane boundary. In machine learning, kernel methods are a class of algorithms for pattern analysis, any linear model can be turned into a non-linear model by applying a non-linear kernel.

Three different kernel functions were tested for classification, Linear Kernel, Gaussian Kernel (radial basis function) and a Polynomial Kernel to analyze which performed better. Kernel is shape of separation boundary- linear/nonlinear boundary represents the similarity of vectors (training samples) in a feature space.

Four types of data sets were analyzed DDC (Direct Data Conversion) in which logical data $(0$ and 1$)$ converted to numerical data (0 and 1), PCA Full (22 feature were reoriented into 22 Principal components), PCA10 / PCARED (22 feature were reoriented into 22 Principal components and 10 were used) and PCA05 (22 feature were reoriented into 22 Principal components and only 05 were used).

The different datasets were used to initially train and afterwards test the SVM algorithm for three different kernels. For the assessment of the performance of classifiers prediction values were calculated for all 3 kernels using all 4 datasets.

PCA Reduced (10 and 5 Principal components) represents the reduction of dimensionality that can be made in two distinctive methods: by just keeping the most applicable factors from the first dataset or by exploiting the redundancy present inherently in the data. For the reduction of data dimensionality principal component analysis (PCA) is used, either using the factors from the first set or using redundancy in the reoriented features [16]. Linear combinations are used to distinguish the variancecovariance structure of an arrangement of factors.

Lastly the predictions were analyzed to assess classifier performance in all cases, the reporting scores of accuracy, precision, sensitivity, specificity and F1-score were estimated through the MATLAB code.

\section{Results}

In total four types of data sets were analyzed for 16 diseases using Support Vector Machine algorithm in MATLAB to check the effect of dimensionality reduction in the original data. The total training labels of all 16 diseases are shown in the table 1 below.

Table 1: Training Samples of all Diseases

\begin{tabular}{|c|c|c|}
\hline \multirow{2}{*}{ Disease } & \multicolumn{2}{|c|}{ Training } \\
\cline { 2 - 3 } & Positive & Negative \\
\hline 1 & 25 & 105 \\
\hline 2 & 41 & 89 \\
\hline 3 & 4 & 126 \\
\hline 4 & 16 & 114 \\
\hline 5 & 28 & 102 \\
\hline 6 & 7 & 123 \\
\hline 7 & 14 & 116 \\
\hline 8 & 4 & 126 \\
\hline 9 & 4 & 126 \\
\hline 10 & 5 & 125 \\
\hline 11 & 5 & 125 \\
\hline 12 & 6 & 124 \\
\hline 13 & 10 & 120 \\
\hline 14 & 5 & 125 \\
\hline
\end{tabular}




\begin{tabular}{|c|c|c|}
\hline 15 & 3 & 127 \\
\hline 16 & 6 & 124 \\
\hline
\end{tabular}

The 4 data sets analyzed were labeled DDC (Direct Data Conversion), PCA22 (All 22 Principal Components used as new features), PCA10 (10 Principal Components were used as new features) and PCA5 (5 Principal Components were used as new features). In DDC, binary data converted to numerical because the SVM Classifier cannot operate on logical data. After this all three kernels Linear, Polynomial (with order 3) and Gaussian were applied to all four types of data separately, kernel is a shape of separation boundary each kernel has different physical/dimensional shape. Accuracy, precision, recall, specificity and $\mathrm{fl}$ score were calculated for performance analysis and results are shown in the following tables $2-4$.

Table 2: Accuracy and Precision for the Linear Kernel

\begin{tabular}{|c|c|c|c|c|c|c|c|c|}
\hline \multirow{2}{*}{ Disease } & \multicolumn{4}{|c|}{ Accuracy } & \multicolumn{4}{|c|}{ Precision } \\
\hline & DDC & PCA 22 & PCA10 & PCA5 & DDC & PCA 22 & PCA 10 & PCA5 \\
\hline 1 & 0.9107 & 0.9107 & 0.8393 & 0.7679 & 0.7778 & 0.7778 & 0.5385 & 0.4118 \\
\hline 2 & 0.9464 & 0.9286 & 0.9464 & 0.9643 & 0.7692 & 0.7143 & 0.7692 & 0.9 \\
\hline 3 & 1 & 1 & 0.9643 & 0.9464 & 1 & 1 & 1 & $\underline{-5}$ \\
\hline 4 & 0.8929 & 0.8929 & 0.8929 & 0.8393 & 0.6667 & 0.6667 & 0.6667 & $-\underline{-}$ \\
\hline 5 & 0.9643 & 0.9643 & 0.9643 & 0.9643 & 1 & 1 & 1 & 1 \\
\hline 6 & 0.9821 & 0.9821 & 0.9286 & 0.9464 & 1 & 1 & 0 & - \\
\hline 7 & 0.8571 & 0.8571 & 0.8571 & 0.8571 & $-\ldots$ & $=$ & $-\ldots$ & $=$ \\
\hline 8 & 0.9643 & 0.9643 & 0.9643 & 0.9643 & $-\overline{-}$ & - & - & $\underline{-}$ \\
\hline 9 & 0.9286 & 0.9286 & 0.9643 & 0.9464 & 0.3333 & 0.3333 & 0.6667 & 0.5 \\
\hline 10 & 0.9464 & 0.9464 & 0.9464 & 0.9464 & -+ & - & $-\infty$ & - \\
\hline 11 & 0.9464 & 0.9464 & 0.9464 & 0.9464 & -- & $\ldots$ & $-\ldots$ & - \\
\hline 12 & 0.9107 & 0.9107 & 0.9464 & 0.9464 & 0.25 & 0.25 & $\overline{-}$ & $-\overline{-}$ \\
\hline 13 & 0.9464 & 0.9464 & 0.9643 & 0.875 & 1 & 1 & 1 & 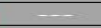 \\
\hline 14 & 0.9643 & 0.9643 & 0.9643 & 0.9643 & 0.5 & 0.5 & $-\overline{-}$ & $-\overline{-}$ \\
\hline 15 & 0.9643 & 0.9643 & 0.9643 & 0.9643 & $-=$ & - & $=$ & $=$ \\
\hline 16 & 0.9643 & 0.9643 & 0.9643 & 0.9643 & $-\overline{-}$ & - & $=$ & -5 \\
\hline
\end{tabular}

Table 3: Recall, Specificity and F1 score values for Linear Kernel

\begin{tabular}{|c|c|c|c|c|c|c|c|c|c|c|c|c|}
\hline \multirow[t]{2}{*}{ Disease } & \multicolumn{4}{|c|}{ Recall } & \multicolumn{4}{|c|}{ Specificity } & \multicolumn{4}{|c|}{ F1 Score } \\
\hline & DOC & $\begin{array}{l}\text { PCA } \\
22\end{array}$ & PCANO & PCA5 & DOC & PCAN22 & PCANO & PCA 5 & DDC & $\begin{array}{l}\text { PCA } \\
22\end{array}$ & $\begin{array}{c}\text { PCA } \\
10\end{array}$ & PCA 5 \\
\hline 1 & 0.7 & 0.7 & 0.7 & 0.7 & 0.9565 & 0.9565 & 0.8696 & 0.7826 & 0.7368 & 0.7368 & 0.6087 & 0.5185 \\
\hline 2 & 1 & 1 & 1 & 0.9 & 0.9348 & 0.913 & 0.9348 & 0.9783 & 0.8696 & 0.8333 & 0.8696 & 0.9 \\
\hline 3 & 1 & 1 & 0.3333 & 0 & 1 & 1 & 1 & 1 & 1 & 1 & 0.5 & \\
\hline 4 & 0.6667 & 0.6667 & 0.6667 & 0 & 0.9362 & 0.9362 & 0.9362 & 1 & 0.6667 & 0.6667 & 0.6667 & \\
\hline 5 & 0.8182 & 0.8182 & 0.8182 & 0.8182 & 1 & 1 & 1 & 1 & 0.9 & 0.9 & 0.9 & 0.9 \\
\hline 6 & 0.6667 & 0.6667 & $D$ & 0 & 1 & 1 & 0.9811 & 1 & 0.8 & 0.8 & - & - \\
\hline 7 & 0 & 0 & 0 & 0 & 1 & 1 & 1 & 1 & $=$ & $\ldots$ & 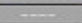 & $\ldots$ \\
\hline 8 & 0 & 0 & 0 & 0 & 1 & 1 & 1 & 1 & $=0$ & $-\infty$ & -3 & $2+$ \\
\hline 9 & 0.3333 & 0.3333 & 0.6667 & 0.3333 & 0.9623 & 0.9623 & 0.9811 & 0.9811 & 0.3333 & 0.33333 & 0.6667 & 0.4 \\
\hline 10 & 0 & 0 & 0 & 0 & 1 & 1 & 1 & 1 & & . & -1 & - \\
\hline 11 & 0 & 0 & 0 & 0 & 1 & 1 & 1 & 1 & & & $=$ & $\ldots$ \\
\hline 12 & 0.3333 & 0.3333 & 0 & 0 & 0.9434 & 0.9434 & 1 & 1 & 0.2857 & 0.2857 & $=$ & $\ldots$ \\
\hline 13 & 0.5714 & 0.5714 & 0.7143 & 0 & 1 & 1 & 1 & 1 & 0.7273 & 0.7273 & 0.8333 & $\ldots$ \\
\hline 14 & 1 & 1 & 0 & 0 & 0.963 & 0.963 & 1 & 1 & 0.6667 & 0.6667 & $-\ldots$ & 20 \\
\hline 15 & 0 & 0 & D & 0 & 1 & 1 & 1 & 1 & - & $\ldots$ & $=$ & $\ldots$ \\
\hline 16 & 0 & 0 & 0 & 0 & 1 & 1 & 1 & 1 & 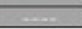 & $+\ldots$ & $\bar{n}$ & $=$ \\
\hline
\end{tabular}

In the case of linear kernel the classifier had different performance for every disease and every condition, some of the diseases showed higher score in DDC data but some has better results with other data types. Each data type shows significant results with overall accuracy of $94 \%, 98 \%$ specificity $69 \%$ F1-score. As expected the dimensionality reduction (PCA22, PCA10 and PCA5) tends towards reduced accuracy however this decrease in performance is really insignificant. The table 4 and 5 below shows the Accuracy and Precision for a Polynomial Kernel with an order of 3.

Table 4: Accuracy and Precision for the Polynomial Kernel

\begin{tabular}{|c|c|c|c|c|c|c|c|c|}
\hline \multirow[t]{2}{*}{ Disease } & \multicolumn{4}{|c|}{ Accuracy } & \multicolumn{4}{|c|}{ Precision } \\
\hline & DDC & PCA 22 & PCA 10 & PCA 5 & DDC & PCA 22 & PCA 10 & PCA 5 \\
\hline 1 & 0.9286 & 0.9286 & 0.8929 & 0.8571 & 0.75 & 0.75 & 0.7 & 0.5833 \\
\hline 2 & 0.9286 & 0.9286 & 0.9464 & 0.9464 & 0.7143 & 0.7143 & 0.7692 & 0.7692 \\
\hline 3 & 1 & 1 & 1 & 1 & 1 & 1 & 1 & 1 \\
\hline 4 & 0.8214 & 0.8214 & 0.8214 & 0.8571 & 0.4 & 0.4 & 0.4 & 1 \\
\hline 5 & 0.9286 & 0.9286 & 0.9643 & 0.9643 & 0.7692 & 0.7692 & 0.8462 & 0.9091 \\
\hline 6 & 0.9464 & 0.9464 & 0.9464 & 0.9464 & $-\cdots$ & 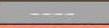 & 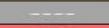 & 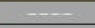 \\
\hline 7 & 0.8036 & 0.8036 & 0.7857 & 0.8571 & 0.2857 & 0.2857 & 0 & -5 \\
\hline 8 & 0.9643 & 0.9643 & 0.9643 & 0.9643 & -1 & -1 & 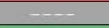 & $-\cdots$ \\
\hline 9 & 0.9643 & 0.9643 & 0.9643 & 0.9464 & 1 & 1 & 1 & 0.5 \\
\hline 10 & 0.9464 & 0.9464 & 0.9464 & 0.9464 & 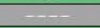 & $=$ & $-\ldots$ & -+- \\
\hline 11 & 0.9643 & 0.9643 & 0.9286 & 0.9464 & 1 & 1 & 0.3333 & $-\ldots$ \\
\hline 12 & 0.9107 & 0.9107 & 0.9464 & 0.9286 & 0.3333 & 0.3333 & 0.5 & 0 \\
\hline 13 & 0.9464 & 0.9464 & 0.9286 & 0.875 & 1 & 1 & 1 & $=$ \\
\hline 14 & 0.9643 & 0.9643 & 0.9643 & 0.9464 & 0.5 & 0.5 & 0.5 & 0 \\
\hline 15 & 0.9643 & 0.9643 & 0.9643 & 0.9643 & 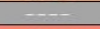 & 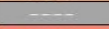 & - & $\overline{-1}$ \\
\hline 16 & 0.9107 & 0.9107 & 0.9286 & 0.9643 & 0.2 & 0.2 & 0.25 & \\
\hline
\end{tabular}


Gyancity Journal of Engineering and Technology,

Vol.5, No.1, pp. 16-25, January 2019

ISSN: 2456-0065 DOI: 10.21058/gjet.2019.51003

Table 5: Recall, Specificity and F1 score values for Polynomial Kernel

\begin{tabular}{|c|c|c|c|c|c|c|c|c|c|c|c|c|}
\hline \multirow[t]{3}{*}{ Disease } & \multicolumn{4}{|c|}{ Recall } & \multicolumn{4}{|c|}{ Specificity } & \multicolumn{4}{|c|}{ F1 Score } \\
\hline & $D D C$ & PCA & PCA & PCA 5 & DOC & PCA 22 & PCA 10 & PCA S & DDC & PCA 22 & PCA 10 & PCA 5 \\
\hline & & 22 & 10 & DLAS & & & & & 0.8182 & 0.8182 & 0.7 & 0.6364 \\
\hline 1 & 0.9 & 0.9 & 0.7 & 0.7 & 0.9348 & 0.9348 & 0.9348 & 0.8913 & 0.8333 & 0.8333 & 0.8696 & 0.8696 \\
\hline 2 & 1 & 1 & 1 & 1 & 0.913 & 0.913 & 0.9348 & 0.9348 & 1 & 1 & 1 & 1 \\
\hline 3 & 1 & 1 & 1 & 1 & 1 & 1 & 1 & 1 & 0.2857 & 0.2857 & 0.2857 & 0.2 \\
\hline 4 & 0.2222 & 0.2222 & 0.2222 & 0.1111 & 0.9362 & 0.9362 & 0.9362 & 1 & 0.8333 & 0.8333 & 0.9167 & 0.9091 \\
\hline 5 & 0.9091 & 0.9091 & 1 & 0.9091 & 0.9333 & 0.9333 & 0.9556 & 0.9778 & & & $\cdots$ & \\
\hline 6 & 0 & 0 & 0 & 0 & 1 & 1 & 1 & 1 & 0.2667 & 0.2667 & $\ldots$ & 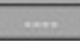 \\
\hline 7 & 0.25 & 0.25 & 0 & 0 & 0.8958 & 0.8958 & 0.9167 & 1 & & - & ... & $\ldots$ \\
\hline 8 & 0 & 0 & 0 & 0 & 1 & 1 & 1 & $\frac{1}{0.011}$ & 0.5 & 0.5 & 0.5 & 0.4 \\
\hline 9 & 0.3333 & 0.3333 & 0.3333 & 0.3333 & 1 & 1 & 1 & 0.9811 & $\ldots$ & $\ldots$ & $\ldots$ & $\ldots$ \\
\hline 10 & 0 & 0 & 0 & 0 & 1 & $\frac{1}{1}$ & 1 & $\frac{1}{1}$ & 0.5 & 0.5 & 0.3333 & $\ldots$ \\
\hline $\begin{array}{l}11 \\
12\end{array}$ & $\frac{0.3333}{0.6667}$ & $\frac{0.3333}{0.6667}$ & $\frac{0.3333}{0.3333}$ & $\frac{0}{0}$ & $\frac{1}{0.9245}$ & $\frac{1}{0.9245}$ & $\begin{array}{l}0.9623 \\
0.9811\end{array}$ & $\frac{1}{0.9811}$ & 0.4444 & 0.4444 & 0.4 & $\ldots$ \\
\hline 13 & 0.5714 & 0.5714 & 0.4286 & 0 & 1 & 1 & 1 & 1 & 0.7273 & 0.7273 & 0.6 & $\cdots$ \\
\hline 14 & 1 & 1 & 1 & 0 & 0.963 & 0.963 & 0.963 & 0.9815 & 0.6667 & 0.6667 & 0.6667 & $\cdots$ \\
\hline 15 & 0 & 0 & 0 & 0 & 1 & 1 & 1 & 1 & $\cdots$ & $\cdots$ & $\cdots$ & $\cdots$ \\
\hline 16 & 0.5 & 0.5 & 0.5 & 0 & 0.9259 & 0.9259 & 0.9444 & 1 & 0.2857 & 0.2857 & 0.3333 & $\ldots$ \\
\hline
\end{tabular}

Lastly the tables 6 through 7 shows the performance of the Gaussian Kernel based SVM.

Table 6: Accuracy and Precision for Gaussian Kernel

\begin{tabular}{|c|c|c|c|c|c|c|c|c|}
\hline \multirow[t]{2}{*}{ Disease } & \multicolumn{4}{|c|}{ Accuracy } & \multicolumn{4}{|c|}{ Precision } \\
\hline & DDC & PCA22 & PCA 10 & PCA 5 & DDC & PCA 22 & PCA 10 & PCA 5 \\
\hline 1 & 0.9643 & 0.9643 & 0.9107 & 0.8036 & 1 & 1 & 0.7778 & 0.4667 \\
\hline 2 & 0.9643 & 0.9643 & 0.9643 & 0.9643 & 0.8333 & 0.8333 & 0.8333 & 0.8333 \\
\hline 3 & 0.9464 & 0.9464 & 0.9464 & 0.9464 & $-\ldots$ & $+\ldots$ & $-\ldots$ & $-\ldots$ \\
\hline 4 & 0.8214 & 0.8214 & 0.8393 & 0.8393 & 0.3333 & 0.3333 & 0.5 & $+\ldots$ \\
\hline 5 & 0.9643 & 0.9643 & 0.9821 & 0.9821 & 0.9091 & 0.9091 & 0.9167 & 1 \\
\hline 6 & 0.9464 & 0.9464 & 0.9464 & 0.9464 & $\ldots$ & $-\ldots$ & $\ldots$ & $-\ldots$ \\
\hline 7 & 0.875 & 0.875 & 0.8571 & 0.8571 & 1 & 1 & $-1+$ & $-1+\ldots$ \\
\hline 8 & 0.9643 & 0.9643 & 0.9643 & 0.9643 & $-\ldots$ & - & $-\cdots$ & $-1+$ \\
\hline 9 & 0.9643 & 0.9643 & 0.9821 & 0.9821 & 1 & 1 & 1 & 1 \\
\hline 10 & 0.9464 & 0.9464 & 0.9464 & 0.9464 & $\ldots$ & $-\ldots$ & $-\ldots$ & $-\ldots$ \\
\hline 11 & 0.9464 & 0.9464 & 0.9464 & 0.9464 & $-\ldots$ & $\ldots$ & $\ldots$ & $-\ldots$ \\
\hline 12 & 0.9464 & 0.9464 & 0.9464 & 0.9464 & 0.5 & 0.5 & $-\ldots$ & $-\ldots$ \\
\hline 13 & 0.9286 & 0.9286 & 0.9464 & 0.875 & 1 & 1 & 1 & $-\overline{-2}$ \\
\hline 14 & 0.9821 & 0.9821 & 0.9643 & 0.9643 & 0.6667 & 0.6667 & $-\ldots$ & 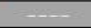 \\
\hline 15 & 0.9643 & 0.9643 & 0.9643 & 0.9643 & $-\ldots$ & 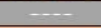 & 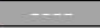 & $-1-$ \\
\hline 16 & 0.9643 & 0.9643 & 0.9643 & 0.9643 & 0.5 & 0.5 & -2 & +2 \\
\hline
\end{tabular}

Table 7: Recall, Specificity and F1score values for Gaussian Kernel

\begin{tabular}{|c|c|c|c|c|c|c|c|c|c|c|c|c|}
\hline \multirow[t]{2}{*}{ Disease } & \multicolumn{4}{|c|}{ Recall } & \multicolumn{4}{|c|}{ Specificity } & \multicolumn{4}{|c|}{ F1 Score } \\
\hline & DDC & PCA22 & PCA10 & PCAS & DDC & PCA22 & PCA10 & PCAS & DDC & PCA22 & PCA10 & PCAS \\
\hline 1 & 0.8 & 0.8 & 0.7 & 0.7 & 1 & 1 & 0.9565 & 0.8261 & 0.8889 & 0.8889 & 0.7368 & 0.56 \\
\hline 2 & 1 & 1 & 1 & 1 & 0.9565 & 0.9565 & 0.9565 & 0.9565 & 0.9091 & 0.9091 & 0.9091 & 0.9091 \\
\hline 3 & 0 & 0 & 0 & 0 & 1 & 1 & 1 & 1 & $\ldots$ & $\ldots$ & $\ldots$ & $\ldots$ \\
\hline 4 & 0.1111 & 0.1111 & 0.2222 & 0 & 0.9574 & 0.9574 & 0.9574 & 1 & 0.1667 & 0.1667 & 0.3077 & $\cdots$ \\
\hline 5 & 0.9091 & 0.9091 & 1 & 0.9091 & 0.9778 & 0.9778 & 0.9778 & 1 & 0.9091 & 0.9091 & 0.9565 & 0.9524 \\
\hline 6 & 0 & 0 & 0 & 0 & 1 & 1 & 1 & 1 & - & $\ldots$ & $\ldots$ & $\ldots$ \\
\hline 7 & 0.125 & 0.125 & 0 & 0 & 1 & 1 & 1 & 1 & 0.2222 & 0.2222 & $\ldots$ & $\cdots$ \\
\hline 8 & 0 & 0 & 0 & 0 & 1 & 1 & 1 & 1 & $\ldots$ & $\ldots$ & $\ldots$ & $\ldots$ \\
\hline 9 & 0.3333 & 0.3333 & 0.6667 & 0.6667 & 1 & 1 & 1 & 1 & 0.5 & 0.5 & 0.8 & 0.8 \\
\hline 10 & 0 & 0 & 0 & 0 & 1 & 1 & 1 & 1 & $\ldots$ & $\ldots$ & $\ldots$ & $\cdots$ \\
\hline 11 & 0 & 0 & 0 & 0 & 1 & 1 & 1 & 1 & $-\ldots$ & $\cdots$ & $\cdots$ & $\cdots$ \\
\hline 12 & 0.3333 & 0.3333 & 0 & 0 & 0.9811 & 0.9811 & 1 & 1 & 0.4 & 0.4 & $\ldots$ & $\ldots$ \\
\hline 13 & 0.4286 & 0.4286 & 0.5714 & 0 & 1 & 1 & 1 & 1 & 0.6 & 0.6 & 0.7273 & $\cdots$ \\
\hline 14 & 1 & 1 & 0 & 0 & 0.9815 & 0.9815 & 1 & 1 & 0.8 & 0.8 & $\ldots$ & $\cdots$ \\
\hline 15 & 0 & 0 & 0 & 0 & 1 & 1 & 1 & 1 & $\ldots$ & $\ldots$ & $\ldots$ & $\ldots$ \\
\hline 16 & 0.5 & 0.5 & 0 & 0 & 0.9815 & 0.9815 & 1 & 1 & 0.5 & 0.5 & $\ldots$ & 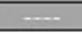 \\
\hline
\end{tabular}

The results were tabulated for both Polynomial and Gaussian Kernels. The Polynomial Kernel based SVM shows an overall average accuracy of across all diseases 93\% and the Gaussian Kernel based SVM shows an overall average accuracy across all diseases of 94\%. Similar to the Linear Kernel, the difference between the different datasets was insignificant for both Polynomial and Gaussian Kernels. 
All diseases have significant result but some of the diseases show extraordinary results because the training data was skewed because the number of positive sample images was far less than the number of negative samples for the disease. This was catered for slightly by the biased random sampling for the training set in the data; however the overall effect of over-fitting is prominent in the data.

This also meant that for some diseases after splitting the data randomly for training and testing the positive samples were significantly reduced which resulted in lack of information.

Hence some of the diseases (e.g. 5 images for disease 15) resulted in heavily biased training. A considerably low number of positive samples in data are the major contributing factor for the low recall scores of all classifiers in all datasets for almost all diseases.

In dimensionality reduction it was noted that as move towards 5 Principal Components instead of 10 or 22 , the accuracy showed a slight decreasing trend but number of false positive increased due to which number of true positive also increased.

\section{Discussion}

Using digital retinal imaging various clinical disorders of the human eye can be examined noninvasively. Automated image analysis can assist in the early detection of a disease based on its earlier symptoms. In order to facilitate the physicians in making better diagnosis, different measurements can be done to detect the retinal diseases quickly and make accurate suggestions based on machine learning algorithms. The aim of this study was to develop an automated tool for classification of retinal diseases in STARE database using SVM classifier in MATLAB, this approach resulted in an average accuracy of $94 \%$.

The proposed algorithm results in significant accuracies for the classification of disease with problems like over-fitting because of biased random sampling for the training data. This also tries to adjust the disproportionate number of positive sample images for some of the diseases. A few diseases were not detected at all due to a heavily biasing training, resulting in wrong classification; it is also expected since the training set consists of 3 positive samples and 127 negative samples for some of the diseases.

The effect of dimensionality reduction on performance was studied, as the system moves towards the lower dimensionality; accuracies generally reduced however this reduction in accuracy was insignificant. Among all three kernels linear, polynomial and gaussian there were insignificant differences between results as gaussian perform just slightly better for our dataset.

On accounts of heavily biased training, resulting in false negative, a problem arose for the diseases with less number of positive samples in the database. This can be improved by applying slightly adjusting the cutoff criterion such as, increasing the number of positive samples (up to 15 instead of 5). This can also be corrected by changing the classifier type or using more features.

Digital image processing techniques have seen remarkable development in the recent years, however for the detection, analysis and treatment of the diseases there is need to further improve the existing methods and develop new ones.

\section{References}

[1] Abràmoff, M. D., Garvin, M. K., \& Sonka, M. Retinal imaging and image analysis. IEEE Reviews in Biomedical Engineering, 3, 169208. (2010).

[2] Rath, P. Contribution of Image Processing and Machine Learning for Automated Analysis of Retinal Vessels : A Review. International Journal of Recent Innovation in Engineering and Research, 2(2), 1-7. (2017).

[3] Nirmala, S. R., Nath, M. K., \& Dandapat, S. Retinal Image Analysis : A Review. International Journal of Computer \& Communication Technology, 2(Vi), 11-15. (2011).

[4] Wu, J., Waldstein, S. M., Montuoro, A., Gerendas, B. S., Langs, G., \& Schmidt-Erfurth, U. Automated Fovea Detection in Spectral Domain Optical Coherence Tomography Scans of Exudative Macular Disease. International Journal of Biomedical Imaging, 1-9. (2016).

[5] Gottlieb, J. L. Age-Related Macular Degeneration. JAMA, 288(18), 2233. (2002).

[6] Singer, D. E., Nathan, D. M., Fogel, H. A., \& Schachat, A. P. Screening for Diabetic Retinopathy. Annals of Internal Medicine, 116(8), 


\section{Gyancity Journal of Engineering and Technology, \\ Vol.5, No.1, pp. 16-25, January 2019 \\ ISSN: 2456-0065 DOI: 10.21058/gjet.2019.51003}

660-671. (1992).

[7] Sowmya, R. A Survey on Automatic Detection of Retinal Disorders from Fundus Images. International Journal of Research in Computer Applications and Robotics, 4(1), 9-15. (2016).

[8] Gharaibeh, N. Y. A Novel Approach for Detection of Microaneurysms in Diabetic Retinopathy Disease from Retinal Fundus Images. Computer and Information Science, 10(1), 1. (2016).

[9] Nasr Y. Gharaibeh. A Novel Approach for Detection of Microaneurisms in Diabetic Retinopathy Disease from Retinal Fundus Images, Computer and Information Science, Vol. 10, No. 1. (2015).

[10]Wu, J., Waldstein, S. M., Montuoro, A., Gerendas, B. S., Langs, G., \& Schmidt-Erfurth, U. Automated Fovea Detection in Spectral Domain Optical Coherence Tomography Scans of Exudative Macular Disease. International Journal of Biomedical Imaging, 1-9. (2016). [11] Priyanka B. Kale, Prof. Nitin Janwe. Detection and Classification of Diabetic Retinopathy in Color Fundus Image, International Journal For Research In Advanced Computer Science And Engineering, Vol. 3, Issue 7. (2017).

[12] Boser, B. E., Guyon, I. M., \& Vapnik, V. N. A Training Algorithm for Optimal Margin Classiiers. Proceedings of the Fifth Annual Workshop on Computational Learning Theory, 144-152. (1992).

[13] The STARE Project. (n.d.). Retrieved August 26, 2018, from http://cecas.clemson.edu/ ahoover/stare/

[14] Karthikram, A., Kavya, P., Keerthika, P., \& Veenmathi, T. Detection of Retinal Diseases Based on SVM Classifier. International Journal of Innovative Research in Computer and Communication Engineering, 3(2), 1548-1554. (2016).

[15] Umesh, L., Mrunalini, M., \& Shinde, D. S. Review of image processing and machine learning techniques for eye disease detection and classification. International Research Journal of Engineering and Technology, 3, 547-551. (2016).

[16] Rasmus Bro, Age K.Smilde. Principal Component Analysis. Anal. Methods. (2014). 